\title{
Study the effect of vibration mixing on the performances of full replacement recycled aggregate concrete
}

\author{
LI Taotao $^{1}$ \\ ${ }^{1}$ CCCC Highway Maintenance Engineering Technology Co., td, 100089, Haidian District, Beijing, China
}

\begin{abstract}
It is an urgent problem that the construction solid waste is difficult to be treated, and its recycling provides a way for its treatment. The quality of construction solid waste recycled aggregate is lower than that of natural aggregate, which limits its extensive application in building materials. In order to provide a new method for engineering application of recycled aggregate concrete, the influence of vibration mixing on the performances of full replacement recycled aggregate concrete were studied. The performance indexes of recycled aggregate concrete under ordinary mixing and vibration mixing were compared and analyzed. The experimental results show that compared with natural aggregate concrete, the slump of full replacement recycled aggregate concrete decreased by $58.8 \%$, and $7 \mathrm{~d}$ and $28 \mathrm{~d}$ compressive strength decreased by $12.9 \%$ and $16.1 \%$, and the splitting strength decreased by $30.6 \%$ and $20.1 \%$, and the carbonation depth decreased by $91.5 \%$; Compared with natural aggregate concrete using ordinary mixing, the slump of full replacement recycled aggregate concrete using vibration mixing decreased by $50.9 \%$, the $7 \mathrm{~d}$ and $28 \mathrm{~d}$ compressive strength decreased by $6.9 \%$ and $10.9 \%$, and the splitting strength decreased by $16.9 \%$ and $12.4 \%$, and the $28 \mathrm{~d}$ carbonation depth decreased by $34.9 \%$. The results show that compared with ordinary mixing, vibration mixing can improve the performance of recycled aggregate concrete, which provides a reference for engineering application.
\end{abstract}

\section{Introduction}

Waste concrete is an important component of building solid waste, which will affect the environment and ecology if it can't be properly disposed. The waste concrete is broken and reused to form recycled aggregate concrete, which provides a way for building solid waste consumption and has positive social significance [1-3]. Takahashi et al. studied the drying shrinkage of recycled coarse aggregate concrete, and found that the drying shrinkage of recycled coarse aggregate concrete is higher than that of natural aggregate concrete, which is related to the mortar ratio [4]. Etxeberria analyzed the possibility of the utilization of recycled concrete aggregates in high performance concrete, and pointed out that $100 \%$ RCA replacement is possible when the aggregates were produced from the original concrete with a minimum compressive strength of $60 \mathrm{MPa}$ [5]. $\mathrm{Li}$ found that the application of recycled fine aggregate reduces the mechanical properties and durability of concrete, and the higher the content of recycled fine aggregate, the more obvious the deterioration of recycled concrete performance [6]. However, due to the weakening of the physical properties of recycled aggregate relative to natural aggregate, the performances of recycled aggregate concrete are also greatly weakened, so it is necessary to improve the performances of recycled concrete in order to be conducive to engineering application. Therefore, strengthening the physical properties of recycled aggregate becomes a way to improve the performance of recycled concrete. Chen used calcium silicate nano gel solution to regenerate aggregate after surface strengthening treatment, and the compressive strength and durability of each age increased greatly [7]. Dong et al. studied the effect of basalt fiber on the mechanical properties of recycled aggregate concrete. The results show that basalt fiber can effectively improve the mechanical properties of recycled aggregate concrete. Compared with the recycled aggregate concrete analysis without adding fiber, the compressive strength and splitting tensile strength are improved by $13.44 \%$ and $6.32 \%$ when the fiber content is $0.3 \%$, which are $39.42 \mathrm{MPa}$ and 3.03 $\mathrm{MPa}$, respectively [8]. The reinforcement of recycled aggregate plays a positive role in improving the performances of recycled concrete, but mixing is an important process of concrete production, and strengthening mixing is also an important way to improve the performances of concrete. Therefore, in this paper, full replacement recycled aggregate concrete is produced using vibration mixing and ordinary vibration technology, and the performances of concrete using two mixing technology compared and analyzed, which provide a new method for improving the performances of recycled aggregate concrete.

\footnotetext{
${ }^{*}$ Corresponding author: 56928514@qq.com
} 


\section{Mechanism of strengthening concrete performance by vibration mixing}

The traditional mixer will do a similar circular motion because of the mixture in the mixing cylinder. Because the mixing shaft has a linear speed when it rotates, it will produce centrifugal force on the mixture in the mixing cylinder. Centrifugal force in mixing cylinder will directly affect the line speed of mixing shaft, and the speed is too high or too low to affect the mixing efficiency and quality. The essence of vibration mixing technology is to apply vibration on the basis of the original mixing, so as to make the mixture vibrate and eliminate the low mixing efficiency area under ordinary mixing, and clean the aggregate surface, seeing the figure 1 . The reason why the micro uniformity of the mixture can be achieved after vibration mixing is that in the mixing process, the vibration of the exciting device is generated by the drive of the motor, which will make the whole mixing device vibrate. The cement paste in the mixture in the mixing tank is impacted by vibration, and the cement that was originally agglomerated together is damaged after impact, so the cement and water can contact together more effectively.

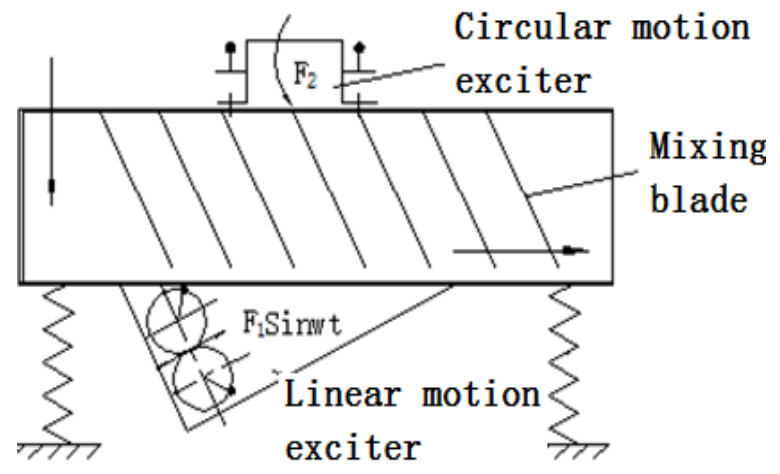

Fig. 1. Simple principle of vibration stirring.

\section{Experimental materials and method}

\subsection{Experimental materials}

The recycled coarse aggregate used in the preparation of concrete specimens is obtained by self-crushing and screening of construction waste after the demolition of a building. The natural sand and aggregate are from a sand and stone factory in Xi'an. Properties of natural and recycled aggregate are shown in table 1. Ordinary portland cement (P.O 42.5, Jidong Cement Jingyang Co., Ltd) is selected for the preparation of concrete, and the water in the process of concrete mixing and hardening is ordinary tap water. According to the concrete proportioning requirements, the aggregate size is 2.36$4.75 \mathrm{~mm}, 4.75-9.5 \mathrm{~mm}, 9.5-19.0 \mathrm{~mm}, 19.0-26.5 \mathrm{~mm}$, and the mass ratio of the four is 3:37:55:5. In the experiment, the replacement rate of recycled aggregate is $100 \%$, seeing table 2 .
Table 1. Properties of natural and recycled aggregate.

\begin{tabular}{ccccc}
\hline Name & $\begin{array}{c}\text { Bulk density } \\
\mathrm{kg} \cdot \mathrm{m}^{-3}\end{array}$ & $\begin{array}{c}\text { Water } \\
\text { absorption }\end{array}$ & $\begin{array}{c}\text { Crushing } \\
\text { value }\end{array}$ & Porosity \\
\hline $\begin{array}{c}\text { Natural } \\
\text { aggregate }\end{array}$ & 2890 & $0.5 \%$ & $5.1 \%$ & $43.5 \%$ \\
$\begin{array}{c}\text { Recycled } \\
\text { aggregate }\end{array}$ & 2320 & $6.7 \%$ & $15.6 \%$ & $48.7 \%$ \\
\hline
\end{tabular}

Table 2. Concrete mix ratio $\left(\mathrm{kg} \cdot \mathrm{m}^{-3}\right)$.

\begin{tabular}{cccccc}
\hline Concrete & Cement & Sand & $\begin{array}{c}\text { Natural } \\
\text { aggregate }\end{array}$ & $\begin{array}{c}\text { Recycled } \\
\text { aggregate }\end{array}$ & Water \\
\hline RAC0 & 351 & 596 & 1268 & 0 & 185 \\
RAC100 & 351 & 596 & 0 & 1268 & 201.6 \\
\hline
\end{tabular}

\subsection{Experimental method}

In this paper, two types of concrete were mixed using the vibration mixer (DT60ZBW type, Xuchang Detong vibration mixing equipment Co., Ltd.). When the vibration mixing is turned on, it is vibration mixing state. When the vibration mixing is turned off, it can complete ordinary static mixing. According to the national standard "GB/T 50080-2016 standard for test methods for performance of ordinary concrete mixture", the samples of the concrete by the two processes were prepared. The slump of rapid carbonation of the fresh concrete and different ages of unconfined compressive strength were tested. The compressive strength test blocks in the experiment were some cubes with a side length of $100 \mathrm{~mm}$. When the samples were cured to the $7 \mathrm{~d}$ and $28 \mathrm{~d}$ ages, they were wiped clean and check their appearance to prepare to be tested. Put the test piece with good appearance in the center of the pressing plate of sans pressure testing machine, and the forming surface is perpendicular to the pressure bearing surface. Then start the testing machine, continuously and evenly load, and record the failure load after the test piece is damaged, seeing fig. 2 .

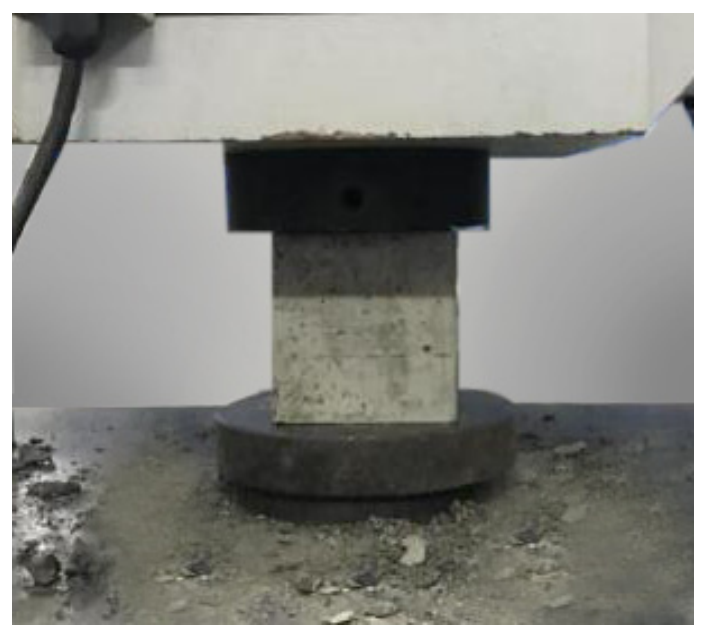

Fig. 2. Compression test of samples. 


\section{Experimental results and analysis}

\subsection{Slump of concrete}

Figure 3 shows the experimental results of slump of full replacement recycled aggregate concrete under two different mixing processes. It can be seen from Figure 3 that the slump of natural aggregate concrete is $51 \mathrm{~mm}$, and that of full replacement recycled aggregate concrete is $21 \mathrm{~mm}$, with a decrease of $58.8 \%$. By using vibration mixing technology, the slump of natural aggregate concrete is $55 \mathrm{~mm}$, and that of full replacement recycled aggregate concrete is $25 \mathrm{~mm}$, which decreases by $54.5 \%$. Compared with ordinary mixing, the slump of natural aggregate concrete increased by $7.84 \%$ and that of full replacement recycled aggregate concrete increased by $19.1 \%$ using vibration mixing process. The experimental results show that the slump of the full replacement recycled aggregate concrete is far lower than that of the natural aggregate concrete, but the slump of full replacement recycled aggregate concrete using vibration mixing is higher than that of the ordinary mixing mixture. The main reason is that although the recycled aggregate has some defects such as high water absorption and micro cracks, which makes the slump of recycled aggregate concrete decrease. The vibration mixing strengthens the arrangement of particles, and increases the hydration reaction of cement, and improves the slump of recycled aggregate concrete.

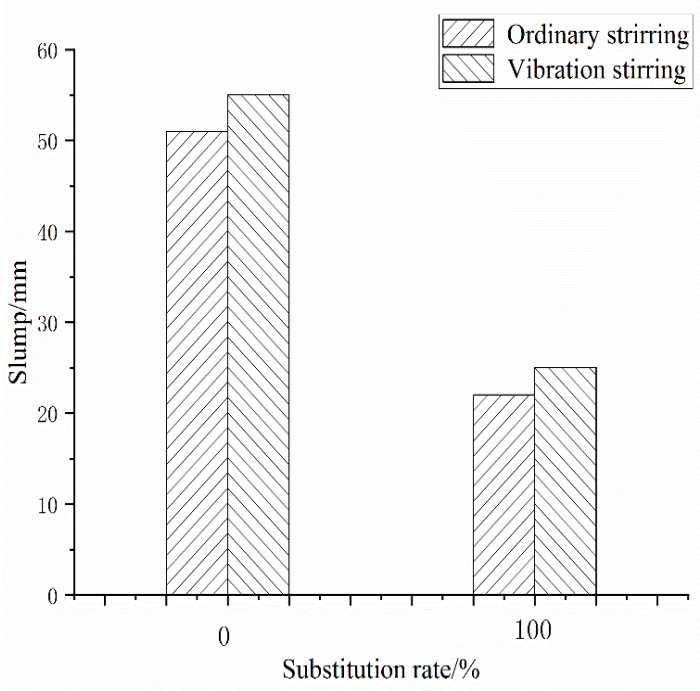

Fig. 3. Slump of full replacement recycled aggregate concrete under two different mixing processes.

\subsection{Compressive strength and splitting strength}

Figure 4 and figure 5 show the experimental results of $7 d$ and $28 \mathrm{~d}$ compressive strength and splitting strength of recycled aggregate concrete with different processes. It can be seen from Figure 4 that, compared with natural aggregate concrete, the $7 \mathrm{~d}$ and $28 \mathrm{~d}$ unconfined compressive strength of full replacement recycled aggregate concrete decreased by $12.9 \%$ and $16.1 \%$ respectively under ordinary mixing process, and decreased by $16.2 \%$ and $19.4 \%$ respectively under vibration mixing process. Similarly, seeing figure 5, compared with natural aggregate concrete, the $7 \mathrm{~d}$ and $28 \mathrm{~d}$ splitting strength of full replacement recycled aggregate concrete decreased by $30.6 \%$ and $20.1 \%$ under ordinary mixing process, and decreased by $22.6 \%$ and $18.8 \%$ under vibration mixing process. Therefore, compared with the ordinary mixing process, the $7 \mathrm{~d}$ and $28 \mathrm{~d}$ unconfined compressive strength of full recycled aggregate concrete is increased by $9.8 \%$ and $8.1 \%$, and the $7 \mathrm{~d}$ and $28 \mathrm{~d}$ the splitting strength are increased by $19.8 \%$ and $9.6 \%$, respectively. The results show that these are caused by the principle of vibration mixing, and vibration mixing can effectively improve the mechanical properties of recycled aggregate concrete by the vibration in the mixing process, and make up for the lack of performances of recycled mixture.

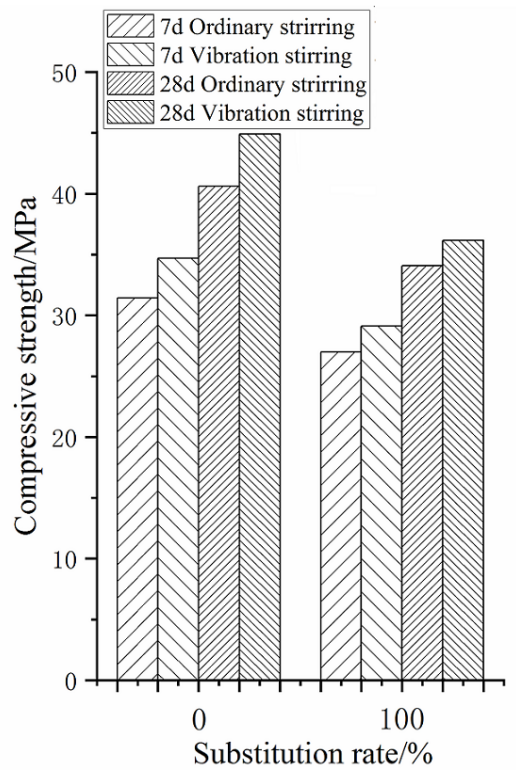

Fig. 4. Compressive strength of recycled aggregate concrete with different processes.

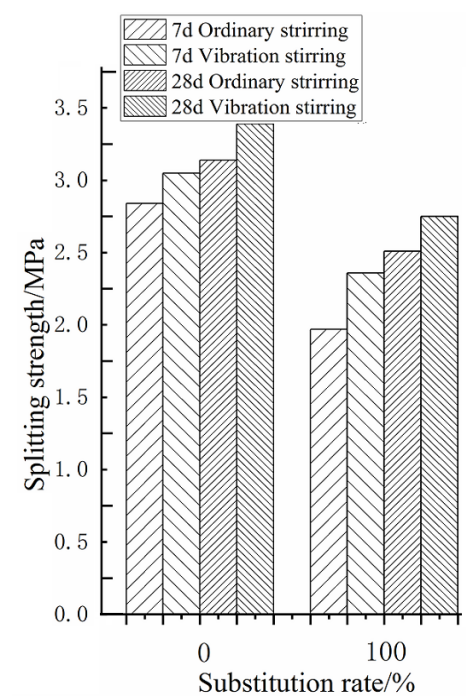

Fig. 5. Splitting strength of recycled aggregate concrete with different processes. 


\subsection{Durability of concrete}

After standard treatment, the samples are put into the concrete rapid carbonation test chamber, when the carbonation age reaches 28 days, the carbonation depth of concrete is tested. Figure 6 shows the experimental results of carbonation depth of recycled aggregate concrete. It can be seen from Figure 6 that, compared with natural aggregate concrete, the carbonation depth of full recycled aggregate concrete under ordinary mixing increases by $91.5 \%$, and that under vibration mixing increases by $34.9 \%$. The experimental results show that the carbonation depth of full recycled aggregate concrete is reduced by $56.6 \%$ after the application of vibration mixing technology, Vibration mixing can greatly improve the durability of recycled concrete.

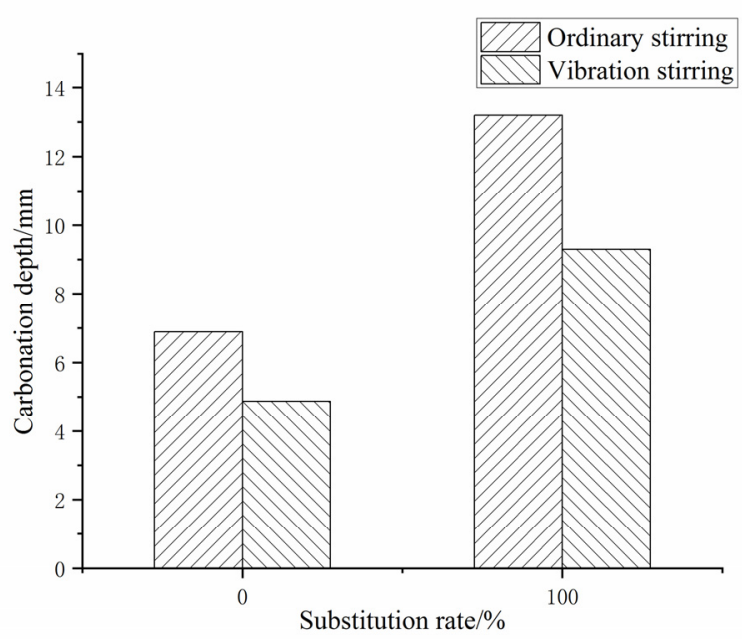

Fig. 6 carbonation depth of recycled aggregate concrete under two mixing processes.

\section{Conclusion}

In order to provide a new method for the large-scale utilization of construction solid waste, realize the recycling of construction solid waste, and alleviate the environmental pollution caused by it. In this paper, the influence of vibration mixing on the performances of recycled aggregate concrete are studied. Through the comparison of vibration mixing and ordinary mixing experiment, the performance indexes of natural aggregate concrete and recycled aggregate concrete under two processes are analyzed. The experimental results show that compared with natural aggregate concrete, the slump of full recycled aggregate concrete under ordinary mixing is reduced by $58.8 \%$, and that under vibration mixing is reduced by $50.9 \%$. The $7 \mathrm{~d}$ and $28 \mathrm{~d}$ unconfined compressive strength of full recycled aggregate concrete decreased by $12.9 \%$ and $16.1 \%$ respectively under ordinary mixing, and decreased by $6.9 \%$ and $10.9 \%$ under vibration mixing. The carbonation depth of full recycled aggregate concrete decreased by $91.5 \%$ under ordinary mixing and decreased by $34.9 \%$ under vibration mixing. Therefore, these results show that the mechanical properties and durability of recycled aggregate concrete can be improved by vibration mixing process, which has engineering application value.

\section{References}

1. X.G. Zhang, H.G. Li, L. Zhu, Concrete, 6 (2015)

2. Y. Obata, R. Yoshida, H. Umehara, Journal of Society of Civil Engineering 70, 2(2014)

3. S.C. Kou, C.S. Poon, Construction and Building Materials, 77(2015)

4. Takahashi Y, Masuda Y, Takeuchi H, Japan Journal of Structural and Construction Engineering 76, 659 (2011)

5. A. Gonzalez, M. Etxebbrria, Construction and Building Materials, 52(2014)

6. J. Sim, C. Park, Waste Management 31, 11 (2011)

7. Chen Weiping, Building Construction 42, 9 (2020)

8. T. DONG, W.J. LIAO, J.J. LIU, X. TANG, F.M. ZOU, H.Y. ZHANG, Concrete, Network publishing $(2020)$ 